\title{
Cooperative Localization in a Distributed Base Station Scenario
}

\author{
Ziming He, Yi Ma, and Rahim Tafazolli \\ Centre for Communication Systems Research \\ University of Surrey, Guildford, UK \\ e-mail:\{z.he, y.ma, r.tafazolli\}@ surrey.ac.uk
}

\begin{abstract}
Previous work about cooperative localization in cellular networks usually consider a centralized processor $(\mathrm{CP})$ is available for location estimation. This paper consider cooperative localization in a distributed base station (BS) scenario, where there is no $\mathrm{CP}$, and the distributed $\mathrm{BSs}$ are responsible for location estimation. In this scenario, Global Positioning System (GPS) enable mobile terminals (MTs), i.e., located MTs, are employed as reference nodes. Then, several located MTs can help to find the locations of an un-located MT, by estimating the distance between the un-located MT using received signal strength techniques. Two localization approaches are proposed, the first approach requires only one BS to collect all the assistance information for localization and estimate the location. The second approach distribute the location estimation task to several BSs. The communication overhead between distributed BSs are investigated for these two approaches. Moreover, by taking into account the effect of imperfect location knowledge of the located MTs, the accuracy limits of both approaches are derived. The simulation results shows that compared with the first approach, the second approach can reduce the communication overhead between distributed BSs with the paid of accuracy.
\end{abstract}

\section{INTRODUCTION}

Cellular network based localization techniques have been extensively investigated over the past decade, e.g., [1]-[6]. Previous approaches includes time-of-arrival (TOA) [1], timedifference-of-arrival (TDOA) [2] and received signal strength (RSS) techniques [3]. The major difference between TOA and TDOA approaches is: TOA approaches require tight clock synchronization between base-station (BS) and mobile terminal (MT), while TDOA approaches only require tight clock synchronization among BSs. Many of the wireless standards only mandate timing clock synchronization among BSs, the MT clock might have a drift of a few microseconds [7]. One of the advantages of RSS approach is that it does not require clock synchronization.

Cooperative localization has been extensively investigated in wireless sensor networks (WSN) [8]-[9]. Recently, the concept of cooperative localization is introduced to cellular networks [10]-[12]. The work in [10] and [12] assume that different MTs can directly communicate with each other in a peer-to-peer (P2P) manner, and clock synchronization is perfect between MTs. Then the distances between MTs is estimated using TOA techniques. Although direct communication between wireless nodes is usually available in WSN, it is not ture for cellular networks. Actually, MTs never communicate directly with each other in cellular networks [11].
A more practical cooperative localization approach for cellular network is proposed in [11]. Their work employs Global Positioning System (GPS) enable MTs (i.e. located MTs) to serve as reference nodes, then the located MTs can help to find the location of an un-located MT. All the assistance information required for location estimation can be forwarded to a centralized processor (e.g. serving radio network controller). In this case, P2P communications are not assumed between MTs. In addition, the distances between located MTs and unlocated MTs are estimated using RSS techniques, thus clock synchronization is not required between MTs. Note that the assistance information includes the location information of the located MTs and RSS-based distance estimates.

From the communication architecture viewpoint, BSs with centralized processor (CP) has several drawbacks. As a network expands, it is expensive to require each new $\mathrm{BS}$ to be connected directly to the CP [13]. Therefore, it is interested to employ distributed BSs, which communicates directly with their neighbors [13]. In distributed BSs scenarios, where a CP is not available, the location estimation can be performed at the distributed BSs. It is of interest to know which BS (or BSs) are going to perform location estimation and how much assistance information is transmitted between BSs. Note that the assistance information for location estimation is overhead for communications. This overhead should be reduced, otherwise it will decrease the efficiency of communication systems.

This paper consider a distributed BS scenario, as depicted in Fig. 1. The serving BS of an un-located MT is the BS in the center, and the serving BSs of several located MTs are the other surrounding BSs. The un-located MT communicates with its home BS on its own uplink frequency, the located MTs listen to this frequency and perform RSS-based distance estimation. Two localization approaches are proposed for the considered scenario. For the first approach, all the assistance information is forwarded to the central BS for location estimation. For the second approach, initial location estimation are performed at the surrounding BSs, receptively. Then the initial estimates are forwarded to the central BS to obtain the final estimate of the un-located MT. Compared with the first approach, the second approach reduce the communication overhead among distributed BSs. However, the second approach may lead to the loss of localization accuracy, due to the fact that the location estimation are distributed among BSs. 


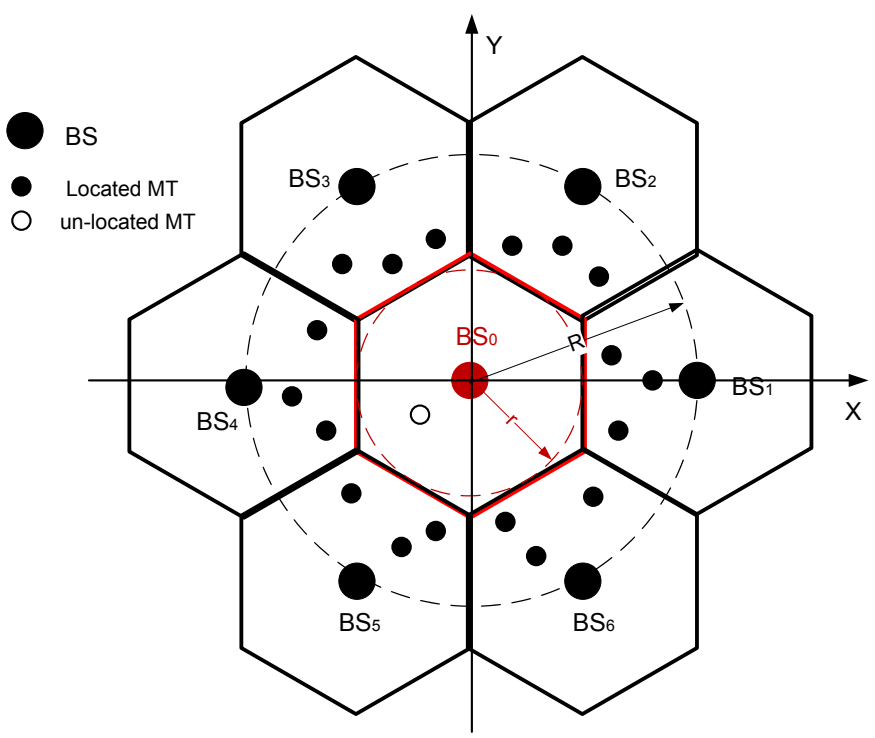

Fig. 1. Scenario and System Model

The major contributions of this paper are: 1) By taking into account the effect of imperfect location knowledge of the located MTs, we derive the accuracy limits of RSSbased cooperative localization in terms of squared position error bound (SPEB) [14]. The derived SPEB can be used to benchmark the accuracy of the approach in [11]. 2) We consider cooperative localization in a distributed BS scenario, which have not been investigated before. Two approaches are proposed for this scenario. Based on 1), the accuracy of these two approaches are derived. Then, the accuracy and communication overhead of these two approaches are compared. Simulation results show that compared with the first approach, the second approach can reduce the communication overhead between distributed BSs with the paid of accuracy.

\section{System Description AND COMMUNiCATION OVERHEAD}

\section{A. System Description}

The system model is depicted in Fig. 1, a seven cells model is considered. The MTs communicate directly with their home BSs, and BSs communicate directly with their neighboring BSs. $\mathrm{BS}_{i}$ is the home BS of the cell with identification (ID) $i$, $i \in[0,6]$. The un-located MT belongs to $\mathrm{BS}_{0}$. The considered located MTs are distributed within any $G$ surrounding cells $(G \in[1,6])$, each of which has $M(M \geq 3)$ located MTs. The total number of located MTs is $N=M G$. The unlocated MT communicates with its home BS on its uplink frequency, which is known by the located MTs. The located MTs listen to this frequency and perform RSS-based distance estimation. Location estimation is performed at BSs after the distance estimates are obtained at the located MTs. Assuming the un-located MT require the location information of itself, the location estimate has to be sent to the un-located MT by $\mathrm{BS}_{0}$ via downlink channel.

\section{B. Communication Overhead}

In order to estimate the location of the un-located MT, assistance information is required. The assistance information for localization includes RSS-based distance estimates and $a$ priori locations knowledge of the located MTs obtained from GPS. Since the a priori locations knowledge is imperfect, it can be treated as random variables with a given probability density function (p.d.f). For simplicity, it is assumed that the $a$ priori locations knowledge of the located MTs are independent Gaussian random variables with the same variance $\omega^{2}$. Then the covariance matrix of the a priori knowledge is a $2 N \times 2 N$ diagonal matrix $\Omega$ with identical diagonal entries $\omega^{2}$. $\omega$ is the accuracy of GPS and have a typical value of $5-10$ (m) [15]. It is reasonable to assume all the BSs knows $\omega$, the located MTs only need to forward the mean of the a priori knowledge to BSs.

For approach 1, all the assistance information is firstly sent to the surrounding BSs by the located MTs. Then, the surrounding $\mathrm{BSs}$ forward it to $\mathrm{BS}_{0}$, which estimate the location and then sent the estimate to the un-located MT via downlink. The communication overhead to forward distance estimates is $32 \mathrm{~N}$ bits (a float requires 32 bits), the overhead to forward the mean of the locations knowledge is $64 N$ bits (2-D location requires two float). Thus, the total overhead between BSs is $96 \mathrm{~N}$ bits for a single location estimation.

For approach 2, when each surrounding BS obtain the assistance information, initial location estimation of the unlocated can be carried out, since $M \geq 3$. Denote $\xi_{g} \triangleq\left(u_{g}, v_{g}\right)$ as the initial location estimate obtained by the $g$-th $(g \in[1, G])$ surrounding BS, $\alpha_{g}^{2}$ and $\beta_{g}^{2}$ as the variance of $u_{g}$ and $v_{g}$, respectively. Assuming $\alpha_{g}^{2}$ and $\beta_{g}^{2}$ can also be obtained at the $g$-th BS, $\xi_{g}, \alpha_{g}^{2}$ and $\beta_{g}^{2}$ are forwarded to $\mathrm{BS}_{0}$. Then approach 2 creates overhead of $32 \times 4 G=128 G$ (bits). At the $\mathrm{BS}_{0}$, the final location estimate $\hat{\theta}$ can be written as a weighting combination of $\xi_{g}$

$$
\hat{\theta}=\left(\mathbf{u}^{T} \mathbf{W}_{\mathbf{u}}, \mathbf{v}^{T} \mathbf{W}_{\mathbf{v}}\right)
$$

where

$$
\begin{aligned}
& \mathbf{W}_{\mathbf{u}}=\frac{\left[\alpha_{G}^{2}, \ldots, \alpha_{1}^{2}\right]^{T}}{\sum_{g=1}^{G} \alpha_{g}^{2}} \\
& \mathbf{W}_{\mathbf{v}}=\frac{\left[\beta_{G}^{2}, \ldots, \beta_{1}^{2}\right]^{T}}{\sum_{g=1}^{G} \beta_{g}^{2}}
\end{aligned}
$$

$\mathbf{u}=\left[u_{1}, \ldots, u_{G}\right]^{T}, \mathbf{v}=\left[v_{1}, \ldots, v_{G}\right]^{T}$,

A summary of communication overhead of these two approaches is shown in Tab I. For a fixed $N$, the maximum overhead of approach 2 is $128 \mathrm{~N} / 3$ (bits) (when $M=3$ ), the minimum overhead is 128 (bits) (when $G=1$ ). Thus, for a single location estimation, approach 2 can reduce at least $160 N / 3$ (bits) and at most $96 N-128$ (bits) compared with approach 1. Obviously, more overhead can be reduced for a larger $N$.

If the un-located MT and located MTs have nearly the same mobility, the located MTs need to continue forwarding their updated locations (i.e. mean of the a priori knowledge) to 
TABLE I

COMMUNICATION OVERHEAD BETWEEN BSS

\begin{tabular}{lr} 
Approach & Overhead (bits) \\
\hline approach 1 & $96 N$ \\
approach 2 & $128 G$
\end{tabular}

their serving BSs for location update of the un-located MT. Then the surrounding BSs employ approach 1 or 2 to forward data to $\mathrm{BS}_{0}$. The overhead is large for successive localization or tracking, thus the overhead reduction using approach 2 is meaningful.

\section{LOCALIZATION ACCURACY}

The localization accuracy of two approaches is analyzed in this section. The accuracy is evaluated in terms of the SPEB defined in [14]. We first derive the SPEB of the approach 1, then the SPEB of the approach 2 can be easily obtained. Note that the SPEB of the approach 1 can be utilized to benchmark the accuracy of the approach in [11], which utilize CP to calculate the location of the un-located MT.

\section{A. SPEB of the approach 1}

Denote $\theta_{n} \triangleq\left(x_{n}, y_{n}\right)$ as the 2-D true location of the $n$-th located MTs $(n \in[1, N]), \theta \triangleq(x, y)$ as the true location of the un-located MT, $p\left(\theta_{1}, \ldots, \theta_{N}\right)$ as the joint p.d.f of the $a$ priori locations knowledge of located MTs, where $x$ and $x_{n}$ denote $\mathrm{X}$ coordinates, and $y$ and $y_{n}$ denote $\mathrm{Y}$ coordinates. The considered problem can be formulated as $N+1$ nodes cooperative localization with a priori location knowledge. The parameters of interests is given by $\boldsymbol{\theta}=\left[\theta_{1}, \ldots, \theta_{N}, \theta\right]^{T}$.

Denotes $\mathbf{P}_{\mathbf{r}}, \mathbf{P}_{\mathbf{t}}, \mathbf{L}$ as $N \times 1$ vectors collect the RSS at located MTs, transmitted power of the un-located MT, and path loss in $\mathrm{dB}$ scale, respectively. $N \times 1$ vectors $\mathbf{e}$ are independent Gaussian random variable representing lognormal fading. Denote $\sigma$ as the standard deviation of the element in e. In a typical case, $\sigma=6-8(\mathrm{~dB})$ [16]. Note that all the entries in $\mathbf{P}_{\mathbf{t}}$ are identical since the un-located MT broadcast training sequence, Then, the following equation holds [17]

$$
\mathbf{P}_{\mathbf{r}}=\mathbf{P}_{\mathbf{t}}-\mathbf{L}+\mathbf{e}
$$

where

$$
\begin{gathered}
\mathbf{L}=10 \gamma \log _{10} \mathbf{d} \\
\mathbf{d}_{n}=\sqrt{\left(x_{n}-x\right)^{2}+\left(y_{n}-y\right)^{2}}
\end{gathered}
$$

$\mathbf{d}_{n}$ denotes the $n$-th element in $\mathbf{d}$.

The Cramér-Rao lower bound (CRLB) with a priori knowledge reads [18, Page 84]

$$
\mathbb{E}_{\mathbf{P}_{\mathbf{r}}, \boldsymbol{\theta}}\left[(\hat{\boldsymbol{\theta}}-\boldsymbol{\theta})(\hat{\boldsymbol{\theta}}-\boldsymbol{\theta})^{T}\right] \geq \mathbf{J}^{-1},
$$

where

$$
\mathbf{J}=\mathbf{J}_{\boldsymbol{\theta}}+\mathbf{J}_{\mathbf{P}}
$$

$\hat{\boldsymbol{\theta}}$ denotes estimates of $\boldsymbol{\theta}, \mathbb{E}_{\mathbf{P}_{r}, \boldsymbol{\theta}}[\cdot]$ the expectation with respect to $\mathbf{P}_{\mathbf{r}}$ and $\boldsymbol{\theta}, \mathbf{J}$ the Fisher information matrix (FIM), $\mathbf{J}_{\boldsymbol{\theta}}$ the FIM from the observations [14] and has the following expression

$$
\mathbf{J}_{\boldsymbol{\theta}}=\mathbb{E}_{\hat{\mathbf{d}}}\left[\frac{\partial}{\partial \boldsymbol{\theta}} \ln p\left(\mathbf{P}_{\mathbf{r}} \mid \boldsymbol{\theta}\right)\left(\frac{\partial}{\partial \boldsymbol{\theta}} \ln p\left(\mathbf{P}_{\mathbf{r}} \mid \boldsymbol{\theta}\right)\right)^{T}\right],
$$

$\mathbf{J}_{\mathbf{P}}$ the FIM from the a priori knowledge [14] and has the following expression

$$
\mathbf{J}_{\mathbf{P}}=\mathbb{E}_{\boldsymbol{\theta}}\left[\frac{\partial}{\partial \boldsymbol{\theta}} \ln p(\boldsymbol{\theta})\left(\frac{\partial}{\partial \boldsymbol{\theta}} \ln p(\boldsymbol{\theta})\right)^{T}\right],
$$

$p(\boldsymbol{\theta})$ the a priori joint p.d.f of $\boldsymbol{\theta} . p(\boldsymbol{\theta})=p\left(\theta_{1}, \ldots, \theta_{N}\right)$, since the un-located MT do not have any a priori location knowledge. According to [19], $\mathbf{J}_{\boldsymbol{\theta}}$ can be written as

$$
\mathbf{J}_{\boldsymbol{\theta}}=\frac{1}{\varepsilon^{2}} \mathbf{H}^{T} \mathbf{H}
$$

where $\mathbf{H}=\nabla_{\boldsymbol{\theta}}^{T} \otimes \mathbf{d}\left(\otimes\right.$ denotes the Kronecker product, $\nabla_{\boldsymbol{\theta}} \triangleq$ $\left.\left[\frac{\partial}{\partial x_{1}}, \frac{\partial}{\partial y_{1}}, \ldots, \frac{\partial}{\partial x_{N}}, \frac{\partial}{\partial y_{N}}, \frac{\partial}{\partial x}, \frac{\partial}{\partial y}\right]^{T}\right), \varepsilon=(\sigma \ln 10) /(10 \gamma), \gamma$ the path loss factor. $\gamma=2$ for free space, $\gamma=4$ is often used to characterize the path loss in urban areas [16]. $\mathbf{H}$ can be further expressed as

$$
\mathbf{H}=\left[\begin{array}{ll}
\mathbf{G} & \mathbf{U}
\end{array}\right]
$$

where the elements of $\mathbf{G}$ and $\mathbf{U}$ are zeros, expect for the following elements

$$
\begin{gathered}
\left\{\begin{array}{l}
\mathbf{G}(n, 2 n-1)=\frac{\cos \phi_{n}}{d_{n}} \\
\mathbf{G}(n, 2 n)=\frac{\sin \phi_{n}}{d_{n}}
\end{array}\right. \\
\left\{\begin{array}{l}
\mathbf{U}(n, 1)=-\frac{\cos \phi_{n}}{d_{n}} \\
\mathbf{U}(n, 2)=-\frac{\sin \phi_{n}}{d_{n}}
\end{array}\right.
\end{gathered}
$$

where $\phi_{n}$ denotes the angle from the $n$-th located MT to the un-located MT, i.e., $\phi_{n}=\tan ^{-1} \frac{y-y_{n}}{x-x_{n}}$. Then (11) can be further written as

$$
\mathbf{J}_{\boldsymbol{\theta}}=\frac{1}{\varepsilon^{2}}\left[\begin{array}{cc}
\mathbf{G}^{T} \mathbf{G} & \mathbf{G}^{T} \mathbf{U} \\
\mathbf{U}^{T} \mathbf{G} & \mathbf{U}^{T} \mathbf{U}
\end{array}\right]
$$

For $2 N$-order Gaussian p.d.f $p\left(\theta_{1}, \ldots, \theta_{N}\right), \mathbf{J}_{\mathbf{P}}$ can be written as [18, Page 85]

$$
\mathbf{J}_{\mathbf{P}}=\left[\begin{array}{cc}
\boldsymbol{\Omega}^{-1} & \mathbf{0} \\
\mathbf{0} & \mathbf{0}
\end{array}\right]
$$

Then

$$
\begin{aligned}
& \mathbf{J}=\left[\begin{array}{cc}
\frac{1}{\varepsilon^{2}} \mathbf{G}^{T} \mathbf{G}+\boldsymbol{\Omega}^{-1} & \frac{1}{\varepsilon^{2}} \mathbf{G}^{T} \mathbf{U} \\
\frac{1}{\varepsilon^{2}} \mathbf{U}^{T} \mathbf{G} & \frac{1}{\varepsilon^{2}} \mathbf{U}^{T} \mathbf{U}
\end{array}\right] \\
& \triangleq\left[\begin{array}{cc}
\mathbf{A} & \mathbf{B} \\
\mathbf{B}^{T} & \mathbf{C}
\end{array}\right]
\end{aligned}
$$

The CRLB of the estimate using approach 1 reads

$$
\mathbf{F}=\left[\mathbf{J}^{-1}\right]_{2 \times 2}=\left(\mathbf{C}-\mathbf{B}^{T} \mathbf{A}^{-1} \mathbf{B}\right)^{-1}
$$

where $\left[\mathbf{J}^{-1}\right]_{2 \times 2}$ denotes the last $2 \times 2$ diagonal submatrix of $\mathbf{J}^{-1}$. According to [14], the SPEB reads

$$
\mathcal{P}=\operatorname{tr}\{\mathbf{F}\}
$$


TABLE II

PARAMETERS FOR SIMULATIONS

\begin{tabular}{lr} 
Parameters & Value \\
\hline$\omega$ & $5(\mathrm{~m})$ \\
$\sigma$ & $6(\mathrm{~dB})$ \\
$\gamma$ & 4 \\
$\mathrm{r}$ & $50(\mathrm{~m})$ \\
$\mathrm{R}$ & $100(\mathrm{~m})$
\end{tabular}

If the location estimation from GPS is perfect, then $\omega=0$, (19) reduce to

$$
\mathcal{P}=\operatorname{tr}\left\{\mathbf{C}^{-1}\right\}
$$

which is the SPEB for single MT localization with $N$ anchors.

\section{B. SPEB of approach 2}

Denote the CRLB matrix for estimation of the un-located MT at the $g$-th surrounding BS as $\mathbf{F}_{\mathbf{g}}$, which can be calculated using (18), then $\alpha_{g}^{2}=\mathbf{F}_{\mathbf{g}}(1,1), \beta_{g}^{2}=\mathbf{F}_{\mathbf{g}}(2,2)$. Assuming $\xi_{g}$ from different surrounding BSs are mutually independent, then the SPEB of the estimate using this approach reads

$$
\mathcal{P}=\mathbf{W}_{\mathbf{u}}{ }^{T} \boldsymbol{\Lambda}_{\boldsymbol{\alpha}} \mathbf{W}_{\mathbf{u}}+\mathbf{W}_{\mathbf{v}}{ }^{T} \boldsymbol{\Lambda}_{\boldsymbol{\beta}} \mathbf{W}_{\mathbf{v}}
$$

where $\boldsymbol{\Lambda}_{\boldsymbol{\alpha}}=\operatorname{diag}\left\{\alpha_{1}^{2}, \ldots, \alpha_{G}^{2}\right\}, \boldsymbol{\Lambda}_{\boldsymbol{\beta}}=\operatorname{diag}\left\{\beta_{1}^{2}, \ldots, \beta_{G}^{2}\right\}$.

\section{Simulation Results and Discussion}

In this section, the simulation results investigate the accuracy of approach 1 and 2 in terms of SPEB. The parameters for simulations are shown in Tab. II. The un-located MTs and located MTs are randomly generated with 10000 realizations. For each realization, the SPEB is calculated using (19), then the empirical cumulative distribution function (c.d.f) of SPEB is plotted. For each realization, the location of the un-located MT is $\theta=\left(\operatorname{real}\left(\rho_{0} e^{j \phi_{0}}\right), \operatorname{imag}\left(\rho_{0} e^{j \phi_{0}}\right)\right)$, the location of the $n$-th located MT is $\theta_{n}=\left(\operatorname{real}\left(\rho_{n} e^{j \phi_{n}}\right), \operatorname{imag}\left(\rho_{n} e^{j \phi_{n}}\right)\right)$. $\rho_{0}$ and $\rho_{n}$ are uniformly distributed within $[0, r]$ and $[r, R]$, respectively. $\phi_{0}$ is uniformly distributed within $[0,2 \pi]$. If the cell ID of the $n$-th located MT is $i(i \in[1,6]), \phi_{n}$ is uniformly distributed within $[\pi(i-1) / 3-\pi / 6, \pi(i-1) / 3+\pi / 6]$.

Fig. 2 investigate the effect of the cell IDs of the located MTs. For the upper plot of Fig. 2, $N=24, G=2$, then $M=$ 12. It is observed that with cell IDs 1 and 4 , the approaches have the best accuracy. The worse appears with cell IDs 1 and 2. The reason is cell 1 and 4 has the largest separated distance and cell IDs 1 and 2 has the smallest. The similar result is observed from the lower plot of Fig. 2, where $N=24$, $G=3, M=8$. The best accuracy appears with cell IDs 1,3 and 5 and the worse appear with cell IDs 1,2 and 3 .

Fig. 3 investigate the effect of $M$ on SPEB with fixed $G$. For the upper plot, $G=2$ with cell IDs 1 and 4 . and for the upper plot, $G=3$ with cell IDs 1,3 and 5 . It is observed that for a fixed $G$, the increase of $M$ leads to the increase of the accuracy, since the total number of located MTs $N$ increases. It is also observed from Tab. I that for a fixed $G$, the overhead for approach 2 is fixed, but the overhead for approach 1 increase with $M$ increases. This indicate that for
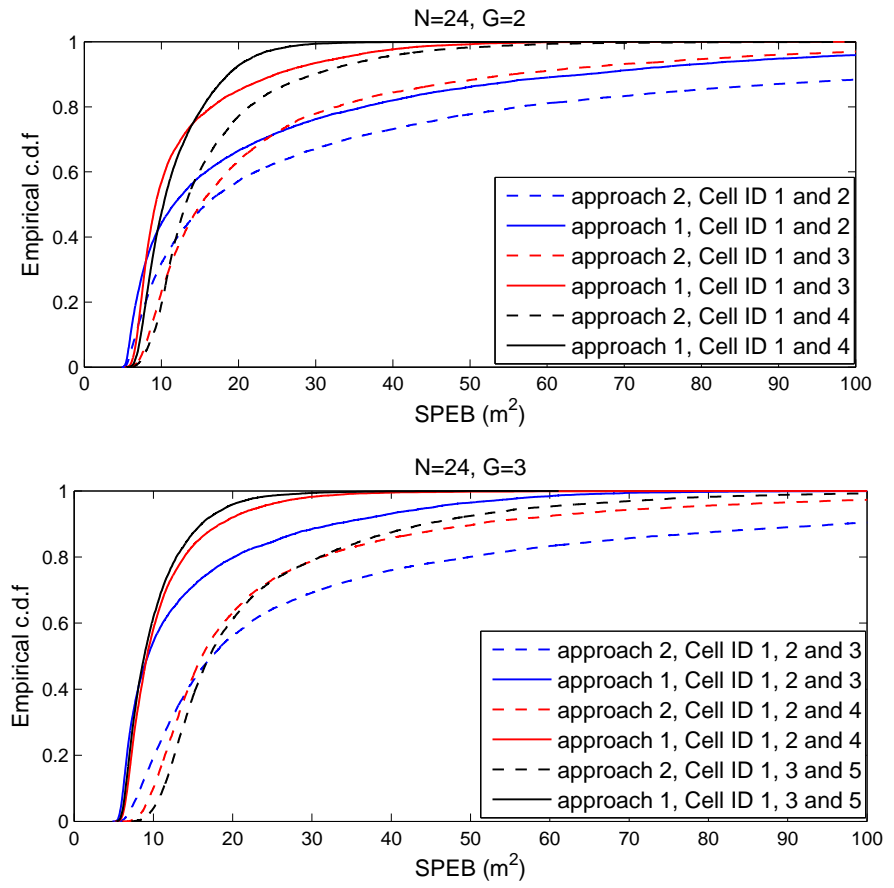

Fig. 2. The effect of the cell IDs

a fixed $G$, approach 2 can reduce more overhead with a larger $M$.

Fig. 4 investigate the effect of $G$ on SPEB with fixed $N$. For fair comparison, the cell IDs are selected in order to achieve the best accuracy, according to the observation in Fig. 2. For $G=2$, the cell IDs are 1 and 4 ; for $G=3$, the cell IDs are 1,3 and 5; for $G=4$, the cell IDs are 1,2,4 and 5; for $G=5$, the cell IDs are $1,2,3,4$ and 5 ; for $G=6$, the cell IDs are $1,2,3,4,5$ and 6 . The effect of $G$ are evaluated with $N=18,24,30,36$, respectively. It is observed from the four subplots that with $G=2$, approach 1 offer slightly worse accuracy than the accuracy with $G=3,4,5,6$. However, approach 2 offer the best accuracy with $G=2$. Thus, the accuracy degradation of approach 2 compared to approach 1 is the smallest with $G=2$. It is also observed from Tab. I that with a fixed $N$, approach 2 can reduce more overhead for a smaller $G$. For the case $G=1$, the two approaches offer the same accuracy, but approach 2 can reduce the overhead by $96 N-128$ (bits).

It is concluded from Fig. 3 and Fig. 4 that approach 1 usually offer better accuracy than approach 2 , but approach 2 outperform approach 1 in terms of communication overhead between BSs. Thus, approach 2 can reduce the overhead the with the paid of accuracy.

\section{Conclusions}

This paper considered cooperative localization in a distributed BS scenario, where there is no CP, and the distributed BSs are responsible for location estimation. In this scenario, the located MTs are employed as reference nodes. Then, 

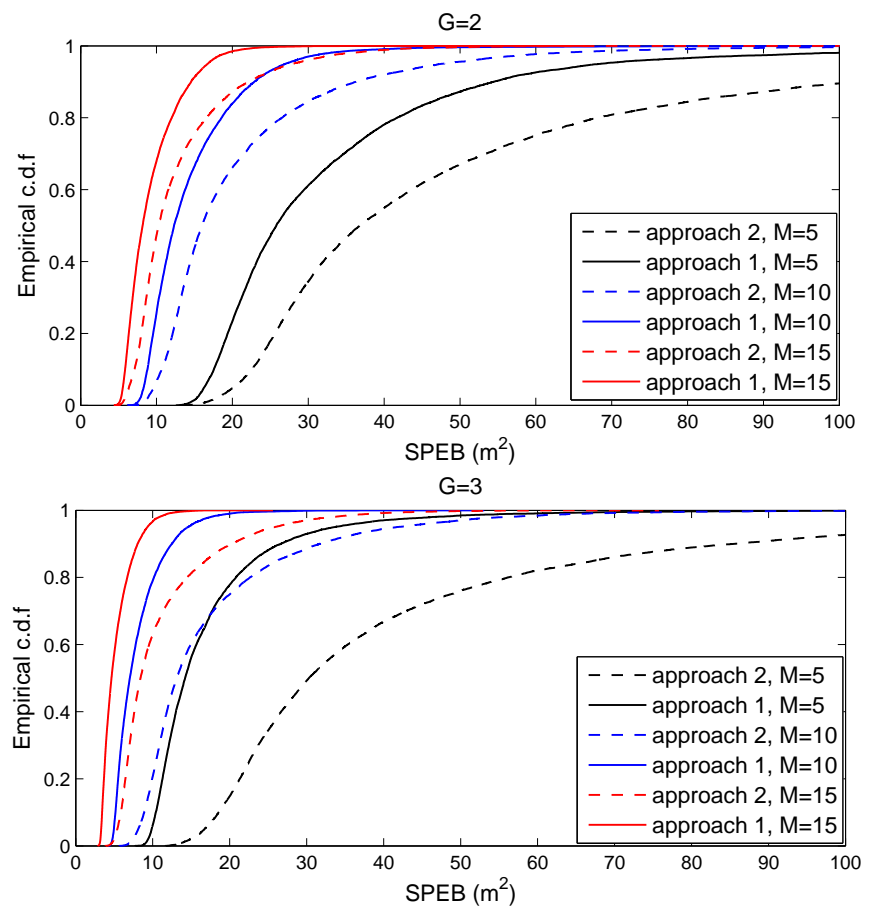

Fig. 3. The effect of the number of located MTs per cell

several located MTs can help to find the locations of an unlocated MT, by estimating the distance between the un-located MT using RSS techniques. We proposed two localization approaches, approach 1 requires only one BS to collect all the assistance information for localization and estimate the location. Approach 2 distribute the location estimation task to several BSs. Moreover, by taking into account the effect of imperfect location knowledge of the located MTs, the accuracy limits of both approaches are derived. The simulation results shown that compared with approach 1, approach 2 can reduce the communication overhead between distributed BSs with the paid of accuracy.

\section{ACKNOWLEDGEMENT}

This work has been performed in the framework of the ICT project ICT- 248894 WHERE 2, which is partly funded by the European Union FP7.

\section{REFERENCES}

[1] J. Caffery and G. L. Stuber, "Subscriber location in CDMA cellular networks", IEEE Trans. Veh. Technol, vol. 47, no. 2, pp. 406-416, May. 1998.

[2] Y. Zhao, "Standardization of mobile phone positioning for 3G systems", IEEE Commun. Mag., vol. 40, pp. 108-116, Jul. 2002.

[3] A. J. Weiss, "On the accuracy of a cellular location system based on RSS measurements", IEEE Trans. Veh. Technol, vol. 52, no. 6, pp. 1508-1518, Nov. 2003.

[4] C. Mensing, S. Sand, A. Dammann, and W. Utschick, "Data-Aided Location Estimation in Cellular OFDM Communications Systems", in Proc. IEEE Global Telecommun. Conf., December 2009.

[5] 3GPP TR 45.811, "Feasibility study on uplink TDOA in GSM and GPRS", http://www.3gpp.org, Jun. 2002.

[6] 3GPP TS 36.305, "Stage 2 functional specifications of UE positioning in E-UTRAN", http://www.3gpp.org, May. 2009.
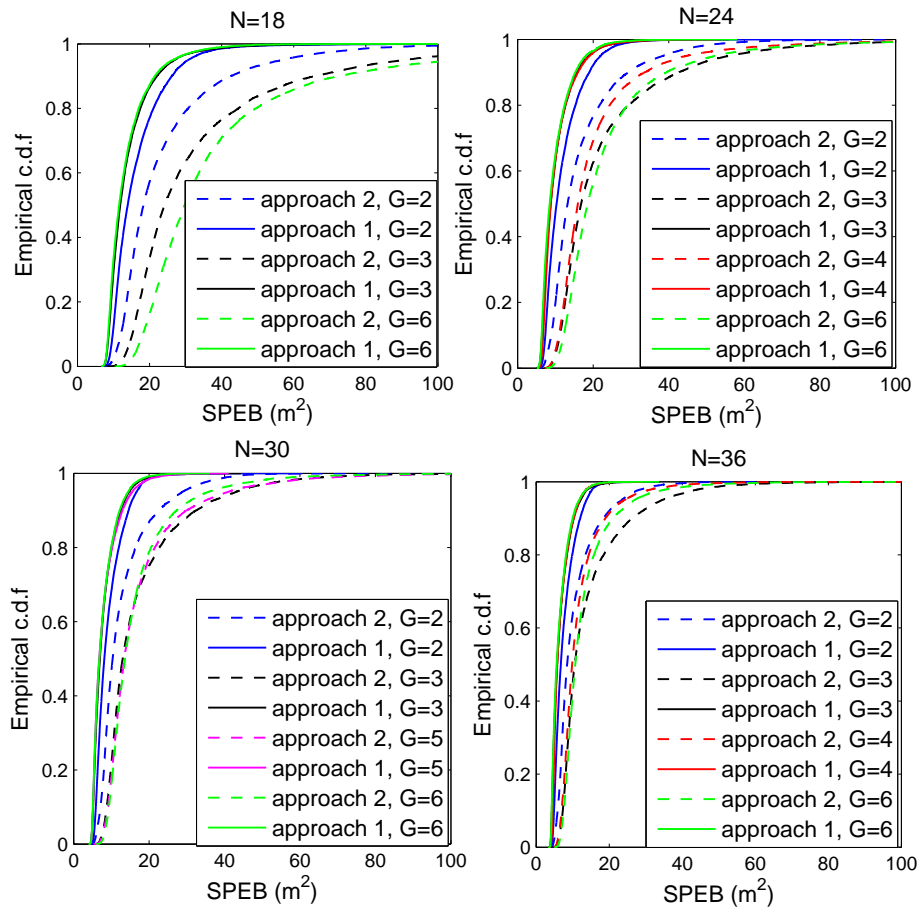

Fig. 4. The effect of the number of surrounding cells that the located MTs belong to

[7] A. H. Sayed, A. Tarighat, and N. Khajehnouri, "Network-based wireless location”, IEEE Signal Process. Mag., vol. 22, no. 4, pp. 12-23, Jul. 2005.

[8] N. Patwari, J. N. Ash, S. Kyperountas, and A. O. Hero III, "Locating the nodes: cooperative localization in wireless sensor networks", IEEE Signal Process. Mag., vol. 22, no. 4, pp. 54-69, Jul. 2005.

[9] G. Mao, B. Fidan, and B. D. O. Anderson, "Wireless sensor network localization techniques", Comput. Netw., vol. 51, no. 10, pp. 2529-2553, Jan. 2007.

[10] S. Frattasi, M. Monti, and R. Prasad, "Cooperative mobile user location for next-generation wireless cellular networks", in Proc. IEEE Int. Conf. Commun., Jun. 2006.

[11] F. L. Piccolo, "A new cooperative localization method for UMTS cellular networks", in Proc. IEEE Global Telecommun. Conf., Dec. 2008.

[12] Y. Zhang, Q. Cui, and X. Tao, "Cooperative group localization for $4 \mathrm{G}$ wireless networks", in Proc. IEEE Veh. Technol. Conf., Apr. 2009.

[13] B. L. Ng, J. S. Evans, S. V. Hanly, and D. Aktas, "Distributed downlink beamforming with cooperative base sations", IEEE Trans. Inf. Theory, vol. 54, no. 12, pp. 5491-5499, Dec. 2008.

[14] Y. Shen and M. Z. Win, "Fundamental limits of wideband localization - part I: A general framework", IEEE Trans. Inf. Theory, vol. 56, no. 10, pp. 4956-4980, Oct. 2010.

[15] F. Gustafsson and F. Gunnarsson, "Mobile positioning using wireless networks", IEEE Signal Process. Mag., vol. 22, no. 4, pp. 41-53, Jul. 2005.

[16] Y. Qi, H. Kobayashi, and H. Suda, "Analysis of wireless geolocation in a non-line-of-sight environment", IEEE Trans. Wireless Commun., vol. 5, no. 3, pp. 672-681, Mar. 2006.

[17] A. Catovic and Z. Sahinoglu, "The cramer-rao bounds of hybird TOA/RSS and TDOA/RSS location estimation schemes", IEEE Commun. Lett., vol. 8, no. 10, pp. 626-628, Oct. 2004.

[18] H. L. Van Trees, Dectection, Estimation, and Modulation Theory-Part I, Hoboken, NJ: Wiley, 1968.

[19] R. W. Ouyang, A. K. S. Wong, and C. T. Lea, "Received signal strengthbased wireless localization via semidefine programming: noncooperative and cooperative schemes", IEEE Trans. Veh. Technol, vol. 59, no. 3, pp. 1307-1318, Mar. 2010. 